\title{
Modelos económico-matemáticos y simulaciones: pilares fundamentales de apoyo a la gestión y la adopción de decisiones en el sector público
}

Nadia Carmen Ene*

Palabras clave: Reformas del sector público, management science, modelos matemáticos, simulación, programas informáticos aplicados, gestión eficiente, investigación operativa.

«La filosofía está escrita en este gran libro -me refiero al universo- que permanece continuamente abierto a nuestras miradas, pero que no puede comprenderse a menos que se aprenda primero a comprender el idioma e interpretar los caracteres en los que está escrito. Está escrito en el lenguaje de las matemáticas, y sus caracteres son triángulos, círculos y otras figuras geométricas, sin los cuales sería bumanamente imposible entender una sola palabra; sin ellos, uno está perdido en un oscuro laberinto».

(Galileo Galilei bablando sobre la modelación de nuestro universo)

\section{La orientación hacia los valores del sector privado en los mode- los de reforma de la Administración Pública}

La orientación del sector público hacia el sector privado, desde el punto de vista de los principios y los métodos de gestión, aparece en los trabajos de referencia de la escuela austriaca de economía, la cual estableció que para convertir el Estado en una organización eficiente era necesario introducir prácticas propias del sector empresarial, como el cálculo de pérdidas y ganancias, la competencia y la descentralización (Von Mises 1932 y Hayek 1944).

* Profesora de Management de la Academia de Estudios Económicos de Bucarest. 
La preocupación por el tema se hace mayor en los años sesenta, cuando en muchos países europeos se comenzó a manifestar la crisis de legitimidad de los poderes públicos como consecuencia de su incapacidad para dar una satisfacción adecuada a las necesidades sociales.

En todos los países, el Estado y las administraciones públicas han sufrido múltiples transformaciones en las últimas décadas debido, en especial, a dos factores. Por una parte, el sector público ha ido asumiendo nuevas funciones en todos los ámbitos de interés social y ha empezado a desarrollar actividades típicamente empresariales, con anterioridad patrimonio exclusivo del sector privado, cada vez más complejas; en estas condiciones, se requieren nuevas formas de gestión en el aparato administrativo. Por otra parte, a largo de los últimos años, las dinámicas de las administraciones públicas de los diferentes países han generado el incremento de su cuota de consumo en el total del PIB, cuestión que ha requerido la introducción en la gestión pública de criterios de eficiencia típicos del sector privado.

En ese mismo contexto, el término globalización es uno de los referentes más utilizados para señalar el origen de muchos de los retos que experimentan en la actualidad Estado y Sociedad. El impacto que ha tenido la globalización económica en las administraciones públicas nacionales a través de los postulados resumidos en el New Public Management (NPM), que ha intentado transformar de manera radical el papel que el Estado ha desempeñado históricamente como garante del desarrollo social y económico de las naciones, representa el mayor desafío al que se ha enfrentado la Administración pública desde que Frederick Taylor y Henri Fayol pregonaron la incorporación de técnicas de gestión a los procesos de gobierno².

En el sentido más estricto de la palabra, el NPM está considerado como un conjunto de dos corrientes de ideas que tienen un origen diferente (Hood 1991: 5). Una hunde sus raíces en la nueva economía institucional que discute nuevos principios administrativos como la competencia, la posibilidad de elección ofrecida a los consumidores, la transparencia y la concentración en estructuras de incentivos. La otra proviene de la aplicación de principios específicos de la gestión del sector privado al sector público.

En efecto, el estudio científico de las actividades de administración y gestión en las organizaciones tanto privadas como públicas ha determinado la aparición de diferentes aportaciones técnicas que han servido para mejorar la eficacia de las organizaciones y se han convertido en disciplinas académicas, dentro de la rúbrica general de técnicas de management. Aunque, en algunos casos, esas técnicas han nacido o se han aplicado por primera vez en la Administración pública (por ejemplo, la técnica PERT de planificación de proyectos, que aparece en la Administración militar de los Estados Unidos), su ámbito de aplicación ha sido básicamente el sector privado.

Las administraciones públicas de muchos países europeos han estado alejadas de esa modernización instrumental o la han iniciado con retraso por varias razones, entre las que cabe destacar el gran peso de la tradición jurídica, como marco comprensivo de la actuación administrativa, o la dificultad para utilizar técnicas desarrolladas en un medio sustancialmente diferente.

De cualquier forma, las administraciones públicas han emprendido un esfuerzo de cimentación de un management público con la incorporación de técnicas que, aun partiendo de hipótesis comunes al management privado, han

\footnotetext{
1 Término aparecido en la literatura española como nueva gestión pública o nueva gerencia pública.

${ }^{2}$ El nuevo estilo administrativo se personalizó en el denominado public management, cuyos principales signos distintivos han sido la prestación de servicios al cliente, el establecimiento de mercados en el seno de los procesos gubernamentales, la orientación empresarial de tipo privada dentro del Estado y la tajante separación entre política y administración.
} 
sido diseñadas para adecuarse a sus necesidades de gestión (por ejemplo, las técnicas presupuestarias por programas o la evaluación de programas públicos). Sin embargo, la introducción de técnicas y métodos específicos del sector privado en la gestión del sector público debe hacerse con cautela, adaptándose a las diferencias entre la gestión pública y privada. Numerosos autores han tratado los factores de especificados de la gestión pública en relación a la gestión empresarial. Así, en el enfoque de Rainey, Backoff, y Levine (1976) se menciona que, junto a los factores ambientales y las operaciones organizacionales, una distinción crítica (muy citada entre las organizaciones del sector público y el privado) consiste en el empleo de métodos distintos para medir la consecución de las metas. Las organizaciones públicas tienen objetivos concurrentes, numerosos y diversos, con criterios vagos para medir el logro de sus objetivos. Los directivos que trabajan en el sector público tienden a tener menos control, flexibilidad y autonomía porque sus subordinados se relacionan con otras autoridades formales para evitar atender las decisiones de sus superiores. Las organizaciones del sector público posen mayor inercia y rigidez y son menos innovadoras en el momento de cambiar. Asimismo, la reducida capacidad de estas organizaciones para concebir y ejecutar planes estratégicos a largo plazo implica también dificultades de cara a la medición de la eficacia.

Una de las facetas más relevantes de cualquier puesto de responsabilidad es la adopción de decisiones. El desarrollo de este proceso dentro del sector público es especialmente complejo. En él toman parte actores con diferentes intereses, orientaciones y sistemas de valores. El proceso de elaboración de las políticas públicas tiende a ser incierto e imprevisible. Muchas veces, los productos y las consecuencias se generan de forma sobrevenida y son resultado fortuito de la interacción entre los marcos de referencia, los valores y las diversas elecciones estratégicas de los actores. Las dificultades de los procesos de elaboración hacen que la preocupación para diseñar herramientas de apoyo y ponerlas a disposición de los decisores sea una constante.

Las barreras generadas por las diferencias arriba enunciadas están ya superadas en la mayoría de los casos cuando se produce la aplicación de una técnica especifica de gestión típica del sector privado en el sector público, siempre que nos refiramos al uso de métodos cualitativos. Pero si miramos hacia el campo de las técnicas de gestión basadas en modelos matemáticos o en el uso de programas informáticos complejos, la brecha entre los dos sectores es todavía muy importante.

En este artículo se analizan las ventajas y posibilidades que ofrecen técnicas y métodos cuantitativos como apoyo a la gestión y la adopción de decisiones en las organizaciones públicas, con objeto de mejorar la eficacia y la eficiencia de las mismas. En las líneas que siguen se presenta el desarrollo científico de la materia, al tiempo que se muestra la evolución de los programas informáticos de modelación y simulación y se describen algunos ejemplos de su aplicación a la esfera de lo público.

\section{La evolución en el campo de la adopción de decisiones a través del uso de modelos matemáticos}

En la segunda mitad del siglo XX empiezan a aplicarse diversos modelos cuantitativos (que combinan conocimientos de matemática, estadística e informática) con el objetivo de mejorar el proceso de toma de decisiones en lo relativo a la rapidez y la obtención de información suplementaria ${ }^{3}$. Las investigaciones operativas

${ }^{3}$ La palabra «Decido», proveniente del latín, tiene dos significados: decidir y también caer. Es por eso que a las plantas a las que se les caen las hojas en otoño se las denomina deciduas. La palabra fall (otoño) era originalmente «leaf falli (caída de las hojas) y se utiliza en lugar 
$(\mathrm{IO})^{4}$ surgieron inicialmente en el contexto militar de la segunda guerra mundial, pero pronto se difundieron a las grandes corporaciones privadas y, hoy en día, se utilizan también en la Administración pública. Para estos fines, han aparecido ramas científicas con varios nombres, dependiendo del país, de la universidad o de la organización que las imparta o aplique: Análisis y Diseño de Sistemas, Investigaciones Operativas, Teoría (o Ciencia) de la Decisión, Modelación y Simulación, Métodos Cuantitativos (en Administración Pública), Ciencia de la Decisión, Management Science ${ }^{5}$, etc.

Las ciencias o asignaturas hasta aquí enunciadas se enmarcan en el enfoque sistémico de solución de problemas. Un elemento importante de este enfoque es la colaboración de distintas disciplinas en la solución de problemas. Esta idea se convirtió en el dogma central de los inicios de la Management Science. Al principio, cuando las Management Sciences llegaron a las organizaciones, los equipos interdisciplinares incluían matemáticos, estadistas, psicólogos, economistas, sociólogos, etc. Con el correr de los años, los equipos interdisciplinares se fueron disolviendo y quienes se incorporaban a la Management Science provenían básicamente de los campos de la matemática y la estadística aplicadas. En el ámbito académico, la Management Science se centró cada vez más en los modelos matemáticos y los algoritmos de solución estratégica, eminentemente rígidos y técnicos. En los últimos años, sin embargo, esta situación ha cambiado con la aparición de metodologías blandas y del pensamiento de sistemas críticos, haciendo posible, de este modo, su aplicación en cualquier tipo de organización.

Mirando atrás en el tiempo, después de la aparición de las investigaciones operativas y hasta un pasado relativamente cercano, numerosos investigadores en el campo de las IO/MS al igual que altos funcionarios del sector público están de acuerdo con la idea de que el impacto y el valor de las IO/MS en el sector público no ha sido tan amplio. Dos son las causas que están detrás de todo ello: (1) algunos fracasos han recibido una amplia publicidad en contraste con la poca difusión que se ha dado a los éxitos registrados en la resolución de algunos problemas muy complejos en el sector público; y (2) el hecho de que muchos de los investigadores en IO/MS no fueran conscientes de que la adopción de decisiones en el sector público es sustancialmente diferente a la que se da en el mundo de los negocios y que, por ello, se hacían necesarias algunas adaptaciones en las técnicas y modelos utilizados.

Hay quienes critican que la introducción de la Management Science en la Administración pública pueda transformarse en una tentativa de sustituir la Ciencia Política tradicional por las ciencias duras y afirman que las ciencias exactas no pueden solucionar los problemas sociales. Otros, subrayan su insuficiencia para ocuparse de la complejidad de los fenómenos sociales y el riesgo de ignorar algunas variables críticas en la búsqueda de determinadas soluciones que aquéllas entrañan. Algunas otras técnicas específicas, como el análisis de costes y beneficios, también han sido criticadas por una variedad de razones, incluyendo su contenido normativo y su excesiva orientación hacia el mercado.

Se ha señalado también que puesto que las dimensiones institucionales y de comportamiento no pueden ser cuantificadas, no pueden ser consideradas en la metodología de la Management Science. A pesar de estos puntos de

de autumn (otoño) en el Siglo XV. La expresión «correr el riesgo» sugiere la importancia de ambos significados. La toma de decisiones equivocadas provoca el miedo a la caída.

${ }^{4}$ Las investigaciones operativas representan un método científico por el cual la administración ejecutiva dispone de una base cuantitativa para las decisiones de operaciones bajo su control. En presente se utiliza también en este contexto el término Operations Research (en E.E.U.U.)/Operational Research (en Gran Bretaña), que se traduce en español por Investigaciones Operativas / Investigaciones Operacionales. Las principales organizaciones a nivel internacional que tratan estos temas son el Institute for Operations Research and the Management Sciences (INFORMS) (www.informs.org) y la Operational Research Society (www.orsoc.org.uk).

${ }_{5}$ Management Science es la ciencia que utiliza la tecnología informática y los modelos matemáticos para ayudar a los actores encargados de ello a adoptar buenas decisiones y a optimizar los procesos organizacionales. Para muchos autores Management Science es una versión más moderna de la investigación operativa. 
vista enfrentados, hay un interés cada vez mayor en las contribuciones de la Management Science como herramientas aplicables a las administraciones públicas.

La aplicación en el sector público de las técnicas cuantitativas de gestión en el proceso decisorio hace necesario que se cumplan determinadas condiciones: a) el respaldo de los funcionarios de alto nivel; b) una asignación clara de las responsabilidades en el proceso de adopción de decisiones; c) la existencia de experiencia con el método utilizado; d) la disponibilidad o la posibilidad de obtención rápida de los datos necesarios; e) y la existencia de registros contables actualizados. Para abordar correctamente la modelación en la toma de decisiones se debe tener una actitud apropiada, además de conocer temas más técnicos. Si bien tanto el analista como el decisor deben conocer las técnicas de identificación de problemas, construcción de modelos y resolución de problemas, las actitudes de ambos quizás constituyan los elementos más importantes para lograr una aplicación exitosa. Una actitud apropiada puede no ser suficiente pero sin duda es una condición necesaria. El analista que se centre más en las técnicas de resolución que en la formulación del modelo no tendrá éxito. El interés principal del analista debiera residir en apoyar la adopción de la decisión y no en hallar métodos de solución que sean más elegantes o más rápidos que los métodos existentes. El decisor que piense que puede dejar trabajar en solitario al analista sin orientación alguna y luego espere obtener información relevante que pueda aplicarse directamente al problema, olvidándose después por completo de lo realizado, no estará haciendo el mejor uso de las aplicaciones cuantitativas. Por contra, la interacción entre el decisor y el analista ha de ser abierta, fluida y focalizada en el objetivo último, que es desarrollar y hacer el mejor uso de las aplicaciones cuantitativas en la resolución de un problema.

Las asignaturas que recurran a métodos cuantitativos y modelos para la Administración pública o para el análisis de políticas públicas deben ser diseñadas de manera que ofrezcan a los estudiantes la oportunidad de desarrollar habilidades analíticas y cuantitativas necesarias para su trabajo profesional. Los cursos se deben centrar en la recogida y la transformación de los datos en información y conocimiento. Un breve análisis de las tendencias curriculares en la enseñanza de la Administración pública en los Estados Unidos indica que existe una tendencia clara en las universidades a formar especialistas en la aplicación de métodos cuantitativos y cualitativos en la gestión y las políticas públicas. Los programas de las asignaturas están concebidos para proveer a los estudiantes de las habilidades gerenciales necesarias para dirigir las organizaciones públicas y de los conocimientos sobre el contexto social, económico y político en el cual se adoptan las decisiones públicas. La Administración pública se percibe desde una perspectiva empresarial y global y se promueve el liderazgo ético. También se reconoce que el éxito gerencial en el sector público requiere de muchas destrezas técnicas.

La situación no es la misma en la universidades europeas, donde la formación es predominante jurídica y eso ya no es suficiente 6 . La constante demanda de mayor eficacia y eficiencia en el sector público han conducido a una redefinición del rol de los funcionarios. Los nuevos funcionarios ahora se caracterizan, además de por su preocupación por garantizar la regularidad de los procedimientos, por su orientación a la acción y a la consecución de resultados, por su capacidad de iniciativa y de asunción de riesgos y por saber negociar y dirigir equipos humanos (Mendoza 1990). Con el desarrollo de las microcomputadoras y de las tecnologías de comunicación de datos, la implantación de sistemas informáticos ha empezado a ocupar una posición esencial en la mejora de la efectividad y eficacia de la administración pública a través de técnicas especificas de management. El avance de la informática, junto con los programas específicos de apoyo al proceso de decisión y gestión, añaden nuevas habilidades a todos los gerentes (tanto en el sector privado como en el público). Los vemos como espíritus jóvenes capa-

\footnotetext{
${ }^{6}$ Chevalier y Loschak (1983) ya constataron que «el administrador jurista se ve substituido por una nueva raza de funcionarios cuya Biblia ya no es el código, sino las consideraciones económicas y sociales»..
} 
ces de utilizar en todos los aspectos de la actividad diaria conocimientos jurídicos, económicos, matemáticos y estadísticos, unidos e incluso potenciados por la tecnología informática.

El uso eficaz de las modernas tecnologías de la información supone una oportunidad para el desarrollo de la Administración, al tiempo que la gestión de los sistemas de información (management of information systems) proporciona una base más objetiva para la toma de decisiones. El uso de sistemas informáticos responde a la necesidad de ayudar a una persona encargada de adoptar decisiones, en definitiva un ser humano con límites específicos, a desarrollar tal tarea. Varios son los límites identificados en tal proceso (Holsapple y Whinston 1996):

- límites cognitivos, que se refieren a la capacidad limitada de los seres humanos de acumular y procesar informaciones y conocimientos;

- límites económicos, relacionados con el coste para obtener y procesar las información y el precio pagado para resolver los problemas de comunicación y coordinación entre los participantes en el proceso de adopción de decisiones;

- límites de tiempo, que se reflejan en los errores y la baja calidad de las decisiones adoptadas bajo la presión del tiempo en un entorno competitivo.

Hoy en día existe una gran cantidad de programas informáticos disponibles para las distintas actividades asociadas a la gestión, que se pueden utilizar o adaptar fácilmente para distintos tipos de procedimientos de adopción de decisiones, algunos específicos del sector público y otros con aplicación tanto en el público como en el privado (como veremos seguidamente).

La modelación en IO/MS ayuda a mejorar las operaciones de empresas y gobiernos mediante el uso de métodos científicos y el desarrollo de técnicas especializadas. La Investigación Operacional no es investigación, es el ciclo del proceso de re-búsqueda de una solución estratégica óptima (o deseable) para el problema/situación de decisión. El proceso de modelación en IO/MS brinda enfoques sistemáticos y generales para solucionar problemas de decisión, cualquiera que sea la naturaleza del sistema, producto o servicio. Los enfoques y las herramientas utilizados en los modelos de IO/CA se basan en los métodos analíticos, la simulación y el razonamiento cualitativo o lógico.

El proceso de modelación en IO/MS es una de las herramientas innovadoras más importantes para la toma de decisiones del siglo XXI. Es un enfoque científico que utiliza escalas mensurables y numéricas para traducir los fenómenos observados. Mediante la aplicación de una aproximación científica, los gerentes también pueden realizar predicciones exactas de lo que no tienen bajo control.

La modelación matemática pretende ser la creación humana más original. Su originalidad reside en el hecho de que los modelos permiten observar conexiones entre las cosas que, a no ser por medio de la razón humana, resultarían extremadamente poco obvias. 


\section{Construir y utilizar modelos matemáticos como herramientas del proceso de gestión y adopción de decisiones}

La adopción de decisiones en las ciencias que usan modelos cuantitativos está basada en tres supuestos: (i) que un problema complejo puede ser descompuesto en pequeñas partes que se pueden analizar más fácilmente, (ii) que las alternativas pueden ser identificadas y evaluadas de forma sencilla y (iii) que el pensamiento sistémico aumenta la posibilidad de obtener mejores decisiones. Otra de sus características distintivas es que los sistemas se pueden representar por modelos en lenguaje simbólico. La expansión de las herramientas informáticas ha facilitado, tanto en la gestión como en el proceso de adopción de decisiones, el uso de estos modelos.

Un modelo es una descripción lógica de cómo se comporta un sistema, proceso o componente de un objeto. Normalmente, su propósito es ayudar a explicar, entender o mejorar un sistema, lo que lo hace útil para la predicción, la deducción y la comunicación, la experimentación, el entrenamiento y la formación (Shannon, 1998).

Algunos ejemplos de modelos utilizados en el sector público (junto con aquellos específicos de la teoría de decisiones) incluyen la programación matemática (lineal y por metas), el método PERT y CPM, la teoría de colas y de redes, la teoría de juegos, los modelos de reemplazamiento, la dinámica markoviana, etc. Debido a que los programas y los proyectos de desarrollo hacen uso con frecuencia del análisis de costes y beneficios, el PERT/CPM ha llegado a ser popular en muchas empresas públicas. La teoría de colas se utiliza mucho en las operaciones portuarias, el transporte por aire y por tierra. Los procesos markovianos se utilizan para la evaluación de recursos humanos en organizaciones, el análisis de programas y políticas sanitarias, la planificación de recursos universitarios, el análisis y predicción de fenómenos de cambio de mercado o en la redistribución de tareas en una organización pública.

En el sector público, la mayoría de las decisiones obligan muchas veces a los decisores a la utilización de varios criterios de decisión para cumplir objetivos contradictorios ${ }^{7}$. Por ejemplo, la decisión sobre el emplazamiento de una sede para una institución pública depende de la infraestructura existente en la región, de las posibilidades de acceso de los ciudadanos pero también de los gastos derivados de la inversión. El estudio de viabilidad sobre una inversión analiza cada opción en términos de costes, periodo de recuperación de la inversión, rendimiento, etc., criterios que se maximizan o se minimizan.

Las organizaciones modernas además, utilizan mucho los grupos para tomar decisiones, sirviendo de ejemplo los Consejos Locales, los equipos de proyecto, o las sesiones de brainstorming. Para facilitar la adopción de decisiones multicriterio y de grupo se han desarrollado herramientas informáticas que permiten la incorporación de un número muy grande de alternativas de decisión. Cabe destacar el programa EXPERT CHOICE de Palisade, que utiliza el análisis jerárquico de Saaty o el HIVIEW y EQUITY de Krysalis, que trabajan con grupos en modo Decision Conference para resolver problemas multicriterio de alta dificultad. Visual Thinking International Ltd. ha desarrollado un programa informático llamado V-I-S-A (Visual Interactive Sensitivity Analysis) que permite la visualiza-

\footnotetext{
${ }^{7}$ Dependiendo de la especificidad del problema que requiere tomar una decisión para alcanzar una solución, se han desarrollado distintos métodos multicriterio para dos categorías de decisiones:

- multiatributo: cuando el número de alternativas es finito, cada alternativa está caracterizada por un número determinado de atributos, y la alternativa preferida es aquélla que satisface lo mejor posible todos los atributos: funciones de utilidad multitributo, analisis jerárquico de Saaty, métodos Electre I y II, etc.

- multiobjetivo: cuando las variables de decisión pueden tomar cualquier valor entre los infinitos posibles delimitados por unas restricciones en forma de igualdades o desigualdades, por ejemplo programación por objetivos (goal programming).
} 
ción explicita de los criterios subjetivos que intervienen en el proceso de decisión. Estos programas ofrecen la posibilidad de incorporar un gran número de criterios de decisión, de asignación de coeficientes de importancia y notas a cualquier nivel, así como escalas de preferencia no lineales. $V$-I-S- $A$ indica a los decisores cuál es la mejor alternativa y cuál es la distancia entre ésta y las otras alternativas de decisión analizadas.

En Hungría, el Departamento y el Laboratorio de Investigaciones Operativas y Sistemas de Decisión del Instituto de Investigaciones sobre Informática y Automatización ha creado un software llamado WINGDSS (Windows Based Group Decision Support System) destinado a apoyar el proceso de adopción de decisiones individuales y de grupo en el sector público. El programa ha sido diseñado con objeto de proveer flexibilidad para detallar los problemas de decisión e incluye un ajustado análisis de sensibilidad y varios instrumentos de visualización. El modelo presupone el conocimiento de tres elementos: las alternativas, los decisores y los atributos de cada alternativa. Los problemas de decisión se resuelven a través de la construcción de un árbol de atributos. Se asigna poder de voto a los decisores y ellos ponderan los atributos y evalúan las alternativas con respecto a los atributos. El programa WINGDSS ha demostrado su viabilidad cuando se ha producido la evaluación de las ofertas realizadas a la Hungarian Power System Operator Company, en relación al análisis de las alternativas para la construcción de la línea 4 de metro en Budapest, y en el desarrollo de una metodología de decisión para evaluar los proyectos de los bancos (por Raiffaisen Bank).

Otro ejemplo de aplicación exitosa lo constituye GASOP, un soporte para las decisiones de grupo que ha demostrado su utilidad en la planificación de la política energética de Indonesia. El Ministerio de Minas y Energía de Indonesia coordina la política energética y su objetivo es el de sostener una combinación energética eficiente desde el punto de vista técnico, financiero y económico para todos los sectores económicos de Java. Antes de GASOP, el Ministerio de Industria indonesio quería utilizar el gas natural como una materia prima barata para algunas industrias energéticas de carácter intensivo (petroquímicas, siderúrgicas, etc.) para incentivar un futuro crecimiento en esos sectores. El Gobierno de Indonesia quería saber en qué mercados, en qué tipo de aplicaciones industriales sería el gas más beneficioso para su economía. Vender el gas a precios altos para las aplicaciones de gran valor conduce a ingresos altos en las ventas domésticas y a una industria doméstica más competitiva mientras que, al venderlo a un precio bajo para las industrias intensivas se inducen ingresos indirectos y el crecimiento futuro de las mismas.

Cada uno de esos objetivos se puede alcanzar por medio de una determinada política energética, pero no ambos al mismo tiempo. Cada alternativa tiene una parte interesada, cada grupo implicado en la planificación de la política energética tiene un interés especial en el asunto y unos conocimientos específicos. Eso conduce a la conclusión de que no existe una solución óptima y única para todos los problemas relacionados. Por eso es necesario realizar un DGS (Decision Group System) que permite a cada subgrupo analizar su sub-problema y formular propuestas teniendo en cuenta las propuestas de los otros grupos con respecto al sub-problema relacionado. Al final es necesario coordinar todas las sub-políticas para formular un plan consistente y comprehensivo. Para eso se ha creado GASOP. La aplicación contiene subsistemas que se pueden utilizar como un DSS o un GDSS, dependiendo de la situación.

\section{Cada módulo del (G)DSS contiene:}

- Un interfaz con el usuario para comunicar los datos de entrada, establecer las variables de decisión e introducir las opciones de política;

- Un sistema de una o más (simulaciones) modelos que describen la situación actual para una cierta área de la política. El sistema se utiliza para evaluar el efecto de las opciones políticas y tiene conexiones con aquellas partes de GASOP que contienen informaciones relevantes.

- Un sistema de salida que permite a los usuarios ver los resultados detallados o en formato grafico. 
Los resultados obtenidos con GASOP han tenido un gran impacto en la formulación de la política energética de Java y se han utilizado para resolver un número importante de problemas en el sector energético.

GASOP ha permitido ordenar las categorías de demanda para la energía. Por primera vez, el Gobierno de Indonesia ha sido consciente de que no era beneficioso servir a todos los consumidores y que es mejor utilizar las reservas escasas de gas de Java en aplicaciones industriales de gran valor. Los resultados de GASOP han servido también para convencer al Gobierno de Indonesia de la necesidad de un ajuste en los precios de la energía en conexión con los costes económicos de los diferentes combustibles y, en consecuencia, de un cambio en la ley de la energía. La aplicación GASOP ha permitido revelar nuevos aspectos de importancia (antes olvidados o desconocidos) de la política energética de Indonesia.

Tradicionalmente, los modelos han sido deterministas. Según estos modelos, no hay ambigüedad en la comprensión del mundo y ningún error en la medida de la evidencia empírica. Este principio tiene sus raíces en la física clásica, pero la tradición determinista de modelos puede conducir a dos riesgos significativos. El primero es el exceso de confianza en las predicciones del modelo. El segundo es el de centrarse más en la precisión que en la exactitud. Para superar el primer riesgo muchos investigadores han utilizado la consideración explícita de una gama de valores del parámetro de entrada con análisis de escenarios. Una minoría de modeladores ha continuado esta trayectoria con la caracterización de las distribuciones del parámetro y la adopción de los análisis sistemáticos de sensibilidad y de incertidumbre.

El grado de abstracción que tienen los modelos matemáticos es un impedimento evidente para su aceptación por parte de los gestores en el sector público. No es de sorprender que existan reticencias entre los gestores que no han recibido suficiente capacitación o exposición a estos modelos y también entre quienes, estando capacitados, no tienen tiempo para prestar la debida atención al modelo. Los modelos matemáticos usan el lenguaje simbólico de las matemáticas, que tiene sus propias limitaciones. Los modelos pueden ser demasiado complejos (como, por ejemplo, el de un aeropuerto internacional) no pudiendo ser resueltos con eficiencia, y requiriendo 'groseras' simplificaciones del problema real para poder llegar a una buena solución estratégica. En tales circunstancias, el problema que queda resuelto ya no se asemeja al problema original y la implantación de la solución puede generar efectos desastrosos en la organización. Una selección apropiada del tipo de modelo y de la técnica de solución debiera minimizar este tipo de error. La mejor alternativa son los modelos de simulación, que son duplicaciones computerizadas de los sistemas reales y, de lejos, son mucho más realistas, en especial en la modelación de sistemas dinámicos y/o probabilísticos.

\section{La simulación como una alternativa más viable a los modelos exactos: evoluciones en el uso de programas informáticas específicos}

El método de simulación para solucionar problemas de optimización está llegando a ser cada vez más popular debido al desarrollo de las TIC y a las varias ventajas de las simulaciones en comparación con los modelos matemáticos exactos. «La mayoría de los modelos matemáticos y estadísticos son estáticos y representan un sistema como un punto fijo en el tiempo. El paso del tiempo no es generalmente un asunto crítico» (Banks 1998: 17), y de manera similar Pidd considera que «la mayoría de los modelos matemáticos no pueden hacer frente satisfactoriamente a los efectos dinámicos o transitorios» (Pidd 1998: 12). 
La simulación implica el diseño del modelo de un sistema, proceso o componente y permite que se ejecuten todos los experimentos posibles en el mismo. El propósito de experimentos tipo ¿Que pasa si...? (What if...?) permite determinar cómo se comportará el sistema real y predecir el efecto de los cambios en el sistema en el transcurso del tiempo.

Para poder decidir correctamente es necesario saber cómo responderá el sistema ante una determinada acción. Esto podría hacerse por experimentación con el mismo sistema, pero factores de coste, seguridad y otros hacen que esta opción generalmente no resulte viable. Para superar estos inconvenientes, se reemplaza el sistema real por otro sistema que en la mayoría de los casos es una versión simplificada. Si bien experimentar con modelos existentes es de lo más común, la simulación permite explorar lo desconocido y no probado. Debido a que la simulación fuerza al modelador a analizar la dinámica del proceso, lo conduce también a un conocimiento más profundo del proceso que está evaluando.

Durante años, las simulaciones han tomado diversas formas, produciendo varias mezclas de hombres y máquinas. Entre éstas, la simulación Montecarlo ${ }^{8}$ es una técnica muy reconocida que combina los conceptos estadísticos (muestreo aleatorio) con la capacidad que tienen los ordenadores para generar números aleatorios y automatizar cálculos. En años posteriores, la simulación Montecarlo se ha venido aplicando a una infinidad de ámbitos como alternativa a los modelos matemáticos exactos o incluso como único medio de estimar soluciones para problemas complejos. Así, en la actualidad es posible encontrar modelos que hacen uso de la simulación Montecarlo en el área informática, empresarial, económica, industrial e incluso pública y social. En otras palabras, la simulación Montecarlo está presente en todos aquellos ámbitos en los que el comportamiento aleatorio o probabilístico desempeña un papel fundamental?.

La simulación es conveniente cuando:

- no existe una formulación matemática analíticamente resoluble o existe una formulación matemática, pero es difícil obtener una solución analítica;

- no existe el sistema real. Es problema del ingeniero que tiene que diseñar un sistema nuevo. El diseño del sistema mejorará notablemente si se cuenta con un modelo adecuado para realizar experimentos;

- los experimentos son imposibles debido a impedimentos económicos, de seguridad, de calidad o éticos. En este caso el sistema real está disponible para realizar experimentos, pero la dificultad de los mismos hace que se descarte esta opción. Un ejemplo de esto es la imposibilidad de provocar fallos en un avión real para evaluar la conducta del piloto, de la misma forma que no se puede variar el valor de un impuesto para evaluar la reacción del mercado;

- el sistema evoluciona muy lentamente o muy rápidamente. Un ejemplo de dinámica lenta es el problema de los científicos que estudian la evolución del clima. Por el contrario, una explosión es un ejemplo de fenómeno muy rápido que debe ser simulado para poder observarlo en detalle.

En el sector público hay muchos procesos donde se pueden utilizar los modelos de simulación, y cabe destacar los procedimientos administrativos. El cuadro 1 presenta las áreas (mencionadas a menudo por el New Public Management) donde se pueden introducir modelos de simulación en la gestión de los procedimientos administrativos.

\footnotetext{
${ }^{8}$ Una «simulación por ordenadon» que supone que el modelo es completamente simbólico, está desarrollada en lenguaje computacional y las personas quedan excluidas del modelo. Los orígenes de esta técnica están ligados al trabajo desarrollado por Stan Ulam y John Von Neumann a finales de los 40 en el laboratorio de Los Alamos (EE.UU.), cuando investigaban el movimiento aleatorio de los neutrones.

${ }_{9}$ Precisamente, el nombre de Montecarlo proviene de la famosa ciudad de Mónaco, donde abundan los casinos de juego y donde el azar, la probabilidad y el comportamiento aleatorio conforman todo un estilo de vida.
} 


\section{CUADRO 1 \\ La utilización de los modelos de simulación en la gestión de los procedimientos administrativos, en vista de los factores integrantes de un servicio}

\begin{tabular}{|c|c|c|c|c|c|}
\hline \multirow[b]{2}{*}{ Funciones } & \multicolumn{5}{|c|}{ Factores en el servicio } \\
\hline & Capacidades & Proveedores & $\begin{array}{c}\text { Actividades- } \\
\text { procedimientos }\end{array}$ & Costes & Usuarios \\
\hline $\begin{array}{l}\text { Planifica } \\
\text { ción }\end{array}$ & $\begin{array}{c}\text { Cálculo de los } \\
\text { equipamientos } \\
\text { necesarios, del grado } \\
\text { de ocupación de las } \\
\text { capacidades }\end{array}$ & $\begin{array}{c}\text { Planificación de } \\
\text { trabajos, } \\
\text { reclutamiento. } \\
\text { Cantidades y } \\
\text { calendarios de los } \\
\text { proveedores }\end{array}$ & $\begin{array}{c}\text { Duración de las } \\
\text { actividades, riesgos } \\
\text { en el cumplimiento } \\
\text { de los } \\
\text { procedimientos }\end{array}$ & $\begin{array}{l}\text { Definición de las } \\
\text { necesidades } \\
\text { presupuestarias, } \\
\text { definición de los } \\
\text { estándares de } \\
\text { costes }\end{array}$ & $\begin{array}{l}\text { Duración de los } \\
\text { procedimientos, } \\
\text { definición de un } \\
\text { horario de trabajo }\end{array}$ \\
\hline Gestión & $\begin{array}{l}\text { Seguimiento del } \\
\text { grado de ocupación } \\
\text { de las capacidades, } \\
\text { de los obstáculos y } \\
\text { su eliminación }\end{array}$ & $\begin{array}{l}\text { Control de la } \\
\text { productividad, } \\
\text { eficiencia y calidad } \\
\text { según las } \\
\text { posiciones } \\
\text { ocupadas y los } \\
\text { centro de costes }\end{array}$ & $\begin{array}{l}\text { Evaluación de los } \\
\text { potenciales } \\
\text { aspectos } \\
\text { inadecuados en los } \\
\text { procedimientos, } \\
\text { obstáculos legales }\end{array}$ & $\begin{array}{l}\text { Seguimiento de los } \\
\text { costes a través de } \\
\text { centros de costes, } \\
\text { poseedores y tipos } \\
\text { de servicios }\end{array}$ & $\begin{array}{l}\text { Información sobre } \\
\text { los responsables de } \\
\text { la realización de los } \\
\text { procedimientos y } \\
\text { sobre los clientes en } \\
\text { uso del servicio }\end{array}$ \\
\hline Análisis & $\begin{array}{l}\text { Análisis del uso de } \\
\text { las capacidades, } \\
\text { evaluación del } \\
\text { realismo de la } \\
\text { planificación, } \\
\text { desviaciones del uso } \\
\text { de las capacidades } \\
\text { del plano inicial }\end{array}$ & $\begin{array}{l}\text { Análisis de } \\
\text { productividad, } \\
\text { eficiencia y } \\
\text { cualidad }\end{array}$ & $\begin{array}{l}\text { Adecuación de los } \\
\text { procedimientos, } \\
\text { simplificación, } \\
\text { complicaciones, } \\
\text { obstáculos legales }\end{array}$ & $\begin{array}{c}\text { Análisis de la } \\
\text { desviación de los } \\
\text { costes reales en } \\
\text { comparación con } \\
\text { los costes } \\
\text { planificados }\end{array}$ & $\begin{array}{l}\text { Igualdad entre los } \\
\text { usuarios, detección } \\
\text { de falta de } \\
\text { integridad en la } \\
\text { gestión } \\
\text { administrativa }\end{array}$ \\
\hline
\end{tabular}

Fuente: Devjak y Pecek, 2002:6.

El proceso de creación de un modelo de simulación se divide en fases empezando con la identificación de la necesidad para crear un modelo de simulación y acabando con las recomendaciones de acuerdo a los resultados del modelo (Filip 2002).

El desarrollo de un modelo de simulación es un proceso complejo y supone (Banks 1998: 28):

- un buen análisis y conocimiento de las características típicas de operación del sistema y de los procedimientos de puesta en práctica del proceso;

- el desarrollo de un modelo de simulación con el ordenador para el sistema estudiado y para sus procesos;

- una prueba sobre la adecuación del modelo de simulación y de una interpretación de los resultados obtenidos.

El desarrollo del modelo de simulación supone utilizar un paquete de software de simulación. Los continuos avances experimentados en las últimas décadas en la informática han dado como resultado una dramática expansión en el uso de la simulación. La tecnología de la simulación ya no está limitada a la existencia de un poderoso hardware y un ingeniero con total dedicación. Hoy, las herramientas disponibles gozan de una sólida reputación, son mucho más amigables e intuitivas, incorporan una importante capacidad gráfica y elementos para facilitar la construcción de simuladores. Con todo ello, en un tiempo prudencial, se puede obtener un modelo con detalles que representen las condiciones asignadas, ofreciendo de esta manera una capacidad de mejora continua para sus operaciones. 
El desarrollo de herramientas informáticas para la simulación ha comenzado en los años 60 y ha conocido un fuerte avance en los años 80 y el principio de los 90 . Este desarrollo ha sido favorecido por la utilización de las aplicaciones de la simulación en el diseño asistido por ordenador y en las decisiones estratégicas de inversiones, en especial en la industria automovilística y los circuitos electrónicos, en la industria aerospacial, en los transportes y en las aplicaciones militares.

Las aplicaciones mencionadas, junto con los progresos en que consiste la tecnología de la información, han tenido un fuerte impacto sobre la arquitectura y las soluciones técnicas adoptadas en la concepción de productos informáticos para la simulación, haciéndolos accesibles para muchas categorías de usuarios poco especializados con el tema de la simulación (especialmente los de la Administración pública). Los avances esenciales se pueden resumir en los siguientes puntos (Nance y Sargent 2002; Jain 1999):

- La utilización cada día mas extendida de los ordenadores personales en la simulación y la integración tecnológica con herramientas típicas para aplicaciones tipo «desktop» (Windows, bases de datos, hojas de calculo electrónico, Visual Basic for Applications - VBA, gráfica de los sistemas de proyección asistida por el ordenador-CAD). El pionero en esta tendencia es el lenguaje SIMAN (incluido actualmente en el instrumento de simulación ARENA, producto de la empresa Rockwel Software);

- La utilización de los efectos visuales, incluidos los de animación y de simulación interactiva visual (Visual Interactive Modeling - VIM, Visual Interactive Simulation - VIS);

- La integración de unos complementos integrados (add-ins) con capacidades avanzadas de optimización junto con aquéllas de simulación. Por ejemplo, ARENA contiene el modulo Opt Quest que permite la identificación y la evaluación de escenarios y alternativas para extremar funciones objetivo con restricciones lineales y expresadas en números enteros. Otro módulo de ARENA, Process Analyzers realiza la comparación entre los modelos después de su elaboración. Otro ejemplo importante es SimRunner Optimization del instrumento Promodel, que permite análisis tipo What if...? y cálculos de optimización.

Muchos autores han apostado por utilizar hojas de cálculo para realizar simulación Montecarlo (Seila 2001; Evans 2000; Gedam y Beaudet 2000). La potencia de las hojas de cálculo reside en su universalidad, en su facilidad de uso, en su capacidad para recalcular valores y, sobre todo, en las posibilidades que ofrece con respecto al análisis de escenarios (what-if análisis).

Las últimas versiones de Excel incorporan, además, un lenguaje de programación propio, el Visual Basic for Applications, con el cual es posible crear auténticas aplicaciones de simulación destinadas al usuario final. En el mercado existen de hecho varios complementos de Excel (Add-Ins) específicamente diseñados para realizar simulación Montecarlo, siendo los más conocidos@Risk y el conjunto de herramientas Decisión Tools Suite (Palisade Corporation $^{10}$ ), Crystall Ball (Decisioneering Inc.), Insight.xla (Dr. Sam Savage Informational Design), SimTools.xla (Roger B. Myerson), etc.

Estas herramientas suponen ciertos conocimientos básicos de estadística (inferencia y probabilidad), así como conocimientos, a nivel de usuario, de la hoja de cálculo Excel. Entre los sistemas informáticos mencionados, los programas integrados en el paquete Decision Tools Suite destacan por su facilidad de utilización, gran rapi-

${ }^{10}$ Fundada en 1984, Palisade Corporation se ha convertido en líder dentro de la industria de software de adopción de decisiones. Desde la introducción de PRISM, la primera aplicación de simulación basada en la simulación Montecarlo para PC, Palisade ha redefinido y extendido las herramientas de software disponibles para ejecutivos y profesionales pertenecientes a las áreas de análisis de riesgo y adopción de

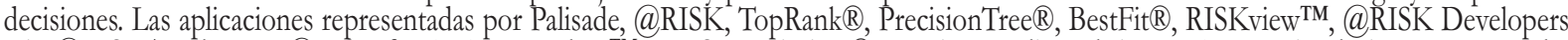
Kit,@RISKAccelerator,@RISK for Project, Evolver ${ }^{\mathrm{TM}}$, y RISKOptimizer ${ }^{\circledR}$ permiten analizar el riesgo, ejecutar simulaciones Montecarlo, realizar análisis de sensibilidad, crear árboles de decisión y diagramas de influencia, ajustar datos a las distribuciones y resolver problemas de optimización. 
dez, potencia de cálculo y fácil navegación entre pantallas, así como, por las posibilidades avanzadas de análisis a través de los resultados presentados en tablas y muchos tipos de gráficos.

Las diferentes ediciones de Decision Tools Suite están formadas por un subconjunto de aplicaciones ([W2]) presentadas brevemente a continuación:

- @RISK es una aplicación avanzada que, mediante el entorno de las hojas de cálculo, permite la modelación del riesgo en todos sus aspectos. Para ello analiza datos, los ajusta a alguna de las 38 funciones de probabilidad disponibles, identifica sus elementos críticos y los escenarios en que actúan, aplica capacidades de simulación avanzadas partiendo de la simulación de Montecarlo y ofrece los resultados tanto a nivel numérico como gráfico para su mejor comprensión e interpretación.

- PrecisionTree utiliza la técnica del árbol de decisión. El árbol de decisión es una técnica muy conocida en el ámbito de la toma de decisiones complejas de un modo lógico y claro, dibujando y visualizando las relaciones entre variables y los nudos de las que dependen. Este programa permite visualizar en diagramas los recorridos de las variables, las opciones y puntos de separación, las influencias de unas sobre otras. Permite, en suma, organizar de modo eficaz toda la información y ver cómo se desarrollan y adónde conducen las diferentes opciones de una decisión. Los proyectos amplios y complejos pueden desglosarse sin perder información ya que permanecen conectados y ayudan a entender la transmisión de las incertidumbres a lo largo de los mismos.

- TopRank es un instrumento para análisis what if...? Esta aplicación identifica los factores críticos de cada decisión, de cada escenario, de cada estrategia y los presenta de modo gráfico, integrando colores, números y formas diferentes para destacar la importancia de cada uno y su contribución a los resultados finales. En conexión con la aplicación base, @RISK proporciona de modo automático y mediante un solo click cuáles son los valores que más afectan a un resultado entre los varios que en ese momento se estén estudiando. Es un programa básico de planificación y juegos de estrategia donde se consideran cientos de posibilidades y opciones.

- BestFit identifica qué tipo de distribución estadística describe mejor los datos históricos o previstos y mediante algoritmos avanzados crea aquellas funciones que pueden ser utilizadas en un formato clásico de hoja de cálculo. Cada grupo de datos en una organización sigue sus propias reglas, es decir, su propio comportamiento probabilístico que muchas veces dista de ser Normal. Tomar decisiones aplicando unas reglas diferentes a las que ordenan los datos históricos o previstos es arriesgado. Esta función es la que realiza de modo instantáneo BestFit en un trabajo que, hasta recientemente, duraba horas.

- RISKview permite visualizar los factores de incertidumbre para estudiar sus formas y características. Riskview es una aplicación cuyas funciones se potencian al ser utilizado conjuntamente con el resto de programas descritos.

- RISKOptimizer permite elegir de entre varias alternativas aquélla que se ajuste mejor a los objetivos que se señalan y busca la solución óptima a los problemas planteados. El programa combina algoritmos genéticos de optimización con la simulación Montecarlo para encontrar la mejor combinación posible de factores que conduzca a un resultado óptimo.

Los proveedores de herramientas informáticas de simulación, los investigadores y los universitarios, consultores y decisores, todos ellos beneficiarios de los modelos de simulación, tienen diferentes opiniones sobre la 
evolución del dominio. Consideran que, en un futuro próximo, van a aumentar las demandas para acortar la duración de los proyectos de simulación y permitir de esta forma tomar decisiones oportunas en tiempo real (Taylor 2000). También, la utilización de la simulación en combinación con el trabajo en equipo asistido por el ordenador y con la tecnología web, facilitará la interacción entre el analista y el encargado para adoptar la decisión y permitirá el funcionamiento eficaz de centros especializados en servicios de simulación (Seila 2001; Taylor 2000). Es de esperar que continúe el proceso de integración de herramientas informáticas en las organizaciones y la elaboración de estándares para ordenar los datos y facilitar el intercambio de informaciones.

Los estudios de simulación son realizados rara vez por un solo analista. En general, son ejecutados por un equipo que reúne todas las habilidades necesarias para el estudio de un sistema complejo. En primer lugar, es necesario disponer de unas personas que entiendan y conozcan el sistema que se está estudiando (los diseñadores del sistema). También es necesaria la participación de los directivos, los líderes de los proyectos y/o el personal operacional que utilizarán los resultados. En segundo lugar, es necesario tener personas que sepan cómo se formula y se modela el sistema, así como programar el modelo (especialistas en simulación) (Shannon 1998). El primer grupo de personas debe ser interno (miembros de la organización para cual se realiza el estudio de simulación). En el caso (muy frecuente en el sector público) de que no se disponga de especialistas de la segunda categoría se puede contratar a personas con las habilidades adecuadas, contratar la modelación a consultores externos, capacitar a personas de la propia organización o combinar ambas opciones.

La diversidad de especialidades que forman parte de un estudio de simulación sugiere la existencia de unas posibles desventajas (dificultades) en lo concerniente a la aplicación de la simulación en la gestión y la adopción de decisiones en el sector público (Banks J. 1998; Morgan y M. Henrion 1990; Shannon 1998):

- el desarrollo de un modelo puede ser costoso, laborioso y lento;

- existe la posibilidad de cometer errores. No se debe olvidar que la experimentación se lleva a cabo con un modelo y no con un sistema real; entonces, si el modelo está mal o se cometen errores en su manejo, los resultados también serán incorrectos;

- no se puede conocer el grado de imprecisión de los resultados. Por lo general, el modelo se utiliza para experimentar situaciones nunca planteadas en el sistema real, por lo tanto no existe información previa para estimar el grado de correspondencia entre la respuesta del modelo y la del sistema real;

- los modelos matemáticos establecidos por ecuaciones complejas serían muy exigentes y también demasiado duros para que un ciudadano ordinario los entendiera o utilizara.

Tal y como se ha señalado anteriormente, las tendencias en el campo del software de simulación hacen que las habilidades de programación y modelación sean menos importantes y mucho más accesibles a la mayoría de los empleados de las organizaciones públicas.

Los métodos cuantitativos para el análisis de políticas públicas en la administración pública se diseñan y se utilizan en las universidades para dar a los estudiantes una ocasión de desarrollar habilidades cuantitativas/analíticas necesarias para su futuro trabajo profesional.

Las simulaciones en el campo del sector público pueden proporcionar a los formadores herramientas muy valiosas para aumentar el conocimiento del estudiante sobre las complejidades del entorno de la realidad de la arena política; mejorar la empatía frente a los desafíos a los cuales se enfrentan los políticos actualmente; y proporcionar vívidas ilustraciones de conceptos teóricos de difícil expresión y comprensión asociados a la adopción de decisiones individuales o de grupo en la gestión de sector publico. 


\section{Consideraciones finales}

Es obvio que los modelos cuantitativos de gestión y adopción de decisiones no pueden constituir una respuesta universalmente válida a los problemas del sector público, pero lo importante es que sí pueden contribuir de modo significativo a la resolución de algunos de esos problemas. La clave está en identificar aquellos ámbitos y procesos de la gestión pública en los que la implantación de esos modelos pueda llegar convertirse en un factor de modernización, que apoye y, a la vez, se apoye en el juicio de las personas como el elemento principal en los procesos de toma de decisiones.

La literatura sobre Administración pública y políticas públicas concede no poca importancia a los modelos formalizados para la adopción de decisiones; sin embargo, las organizaciones públicas se enfrentan habitualmente con limitaciones a la hora de aplicarlos: de formación, tiempo, recursos, fuentes de información y también de competencias.

Los métodos vinculados a la investigación operativa, el análisis de sistemas y la teoría de la decisión estadística son especialmente útiles en la adopción de decisiones racionales/estructuradas, aquéllas que se asocian a los niveles inferiores de la gestión. En cambio, en la adopción de decisiones estratégicas, las que se desarrollan en niveles directivos, la simulación ofrece una alternativa más viable por su flexibilidad y posibilidades de aplicación en medios caracterizados por el riesgo y la incertidumbre.

En esencia, nos encontramos dos visiones alternativas sobre el uso de los modelos cuantitativos en la gestión. En una de ellas, se acepta que, si se usan apropiadamente, esos modelos darán la única respuesta correcta al problema y prescribirán el curso de acción que tendrá que seguir el decisor. Desde la otra visión, se considera que los modelos son natural y esencialmente inútiles; el proverbial fuego fatuo, y por ello las personas «prácticas» no debieran perder su tiempo estudiándolos. La verdad acaso se encuentre entre estas dos opiniones extremas. Los modelos cuantitativos pueden sernos útiles si logramos encontrar el momento y el lugar correctos en el que utilizarlos en el análisis de problemas y la toma de decisiones.

En efecto, los modelos cuantitativos aplicados en la Administración pública permiten, en no pocas ocasiones, pasar de la intuición y falta de experiencia, de la falta de objetivos o de formulaciones imprecisas, de la información reducida y parcial, del recurso a la prueba y error o de las predicciones sobre el futuro a través del pasado, a la identificación de las relaciones causa-efecto, a una información rápida y adecuada, a la investigación de fenómenos complejos y a la formulación de objetivos claros y a posibilitar el seguimiento del logro de los mismos.

El marco actual y los retos que se le plantean al Estado y a sus administraciones amplía la libertad de las mismas para explorar la utilización de nuevos métodos que permitan una gestión más eficaz, más eficiente y de mayor calidad. Así, los proveedores de servicios públicos deben ofrecer a los usuarios información apropiada sobre la tramitación de sus asuntos. No se olviden tampoco las posibilidades que con ello se abren para la obtención de información en tiempo real sobre todo el curso de la acción. Otra de las ventajas del uso de modelos cuantitativos en la Administración consiste en la posibilidad de almacenar la información de forma más sistemática y mejorar las condiciones de acceso a las bases de datos, lo que resulta particularmente atractivo desde el punto de vista de la reducción de costes. Todo ello nos sitúa acaso un paso más cerca de los modelos eficientes de control y supervisión del sector privado. En cualquier caso, lo más importante es que no sólo estamos abocados a redefinir los procedimientos y hasta los contornos de las organizaciones públicas sino también a contar con unos medios y unas aplicaciones informáticas adecuadas, de tal modo que la gestión y la toma de decisiones puedan verse real y eficientemente apoyadas por el uso de modelos matemáticos. 
Una última observación. En un mundo en el que emerge con fuerza la equidad como criterio de valor en la gestión, la utilización de los modelos cuantitativos puede contribuir de forma significativa a promover la igualdad de trato e incluso a reducir la corrupción, al uniformizar los procedimientos en las distintas unidades administrativas. En la medida en que ello se consiga, se confirmaría definitivamente que dichos modelos constituyen herramientas fundamentales de apoyo a una gestión congruente con los nuevos marcos de gobernanza.

\section{REFERENCIAS BIBLIOGRÁFICAS:}

Banks, J. (1998), Hanbook of Simulation. Nueva York: John Wiley \& Sons.

Barba-Romero S. (1984), Técnicas de Apoyo a la Toma de Decisiones en la Administración Pública. Madrid: Instituto Nacional de Administración Pública.

Barrera Grageda, J. M. (2004), «¿El fin de la administración pública?», Economia, Sociedad y Territorio IV 16: 747-764.

Bates, J.G. (1993), Managing value for money in the public sector. London: Chapman \& Hall.

Camm, J.D. y Evans, J.R. (1996), Management Science: Modelling, Analysis and Interpretation. South - Western College Publishing.

Chevalier, J. y Loschak, D. (1983), Ciencia Administrativa. Madrid: Instituto Nacional de Administración Pública.

Devjak, S. y Peèek B. (2002), «Simulation Techniques in Public Administration Procedures», International Symposium on Learning Management and Technology Development in the Information and Internet Age. The convergent paths of Public and Private Organizations, University of Bologna.

Dowlatabadi, H., Lawrence, H. G. y Raymond, J. K. (1994), Integrated Economic and Ecological Modeling for Public Policy Decision Making, Final Report Prepared for Adaptation Branch, Climate Change Division Economic Analysis \& Research Branch, Economic Analysis \& Innovation Division Office of Policy, Planning and Evaluation U.S. Environmental Protection Agency Washington D.C., <http://www.rff.org/ kopp/Reports/meta_modeling_report.pdf >.

Evans, J.R. (2000), «Spreadsheets as a Tool for Teaching Simulation», Informs Transactions On Education 1. < http://ite.informs.org / Vol1No1/evans/evans.html>

Filip, G.F. (2002). Decizie asistata de calculator. Decizii, decidenti, metode si instrumente de bazã. Bucuresti: Editura Tehnica \& Editura Expert.

Gedam, S.G. y Beaudet, S.T. (2000), «Monte Carlo Simulation using Excel Spreadsheet for Predicting Reliability of a Complex System», Proceedings Annual Reliability and Maintainability Symposium.

Hayek, F.A. (1944), The Road to Serfdom. London: George Routledge Son.

Holsapple, C.V. y Whinston, A.B. (1996), Decision Support Systems: A Knowledge-Based Approach. Mineapolis/St. Paul: West Publishing Company,

Hood, C. (1996), «Contemporary Public Management: A New Global Paradigm?», Public Policy and Administration 10(2): 104-117. Hood, C. (1996), «A Public Management for All Seasons», Public Administration., 69-1: 3-19.

Jain, S.M. (1999), «Simulation in the Next Millennium» en P.A., Farrington, D.T., Nembhard, D.T. Sturrock y G.W. Evans eds., Proceedings of the 1999 Winter Simulation Conference.

Mendoza, X. (1990), «Técnicas gerenciales y modernización de la Administración Pública en España», Documentación Administrativa: Técnicas gerenciales en la Administración Pública 223. 
Mises, L. (1932), Die Gemeinwirtschaft. Jena: Gustav Fischer.

Morgan, M.G. y Henrion M. (1990), Uncertainty: A Guide to Dealing With Uncertainty in Quantitative Risk and Policy Análisis. Nueva York: Cambridge University Press.

Nance, R.E. y Sargent, R.G. (2002), «Perspectives on the Evolution of Simulation», Operations Research 50-1: 161-172.

Ocampo, B. (2000), «Models of Public Administration Reform: "New Public Management (NPM)"», Asian Review of Public Administration XII - 1: 248-255.

Patterson A., y O’Reilly L.G. (2002), «Simulation and Modeling: A Tool for Public Policy Research», International Association of Maritime Universities Journal.

Pidd, M. (1998), Computer Simulation in Management Science. New York: John Wiley \& Sons.

Rainey, H.G., Backoff, R.W. y Levine, C.H. (1976), «Comparing public and private organizations», Public Administration Review 36: 233-244.

Seila, A.F. (2001), «Spreadsheet Simulation», Proceedings of the 2001 Winter Simulation Conference $74-78$.

Shannon, R. (1998), «Introduction to the art and science of simulation», en D.J. Medeiros, et al. (1998), Proceedings of the 1998 Winter Simulation Conference.

Taylor, S.J.E. (2000). «Groupware and the simulation consultant», en S. Andradottir, et al. (1997), Proceedings of the 1997 Winter Simulation Conference.

United Nations. Division for Public Economics and Public Administration, Department of Economic and Social Affairs, Retbinking Public Administration: An Overview, Governance and Public Administration Branch (GPAB), Division of Public Administration and Development Management (DPADM), Department for Economic and Social Affairs (DESA).

\section{Recursos on line}

Breve historia de los orígenes del método Monte Carlo. <http://www.geocities.com/CollegePark/Quad/2435/index.html>

Decision Tools Suite <http://www.palisade.com/>

Crystal Ball <http://www.crystalball.com/>

Breve artículo sobre simulación MC con Excel <http://www.projectware.com.au/tutorials/Tu08.pdf:>

Hossein ARSHAM, Ciencia de la Administración Aplicada para Gerentes y Lideres Gerenciales: Toma de decisiones estratégicas acertadas. $<$ http://www.ubmail.ubalt.edu/harsham:> 


\section{Experiencias y Casos}

